\title{
盘式永磁调速器在离心负载下的调速性能*
}

\author{
吴 明 1,2 马玉顺 1,2 匡 俊 1,2 黄晓兵 ${ }^{2}$ \\ (1. 中船重工第 702 研究所 上海 200011; \\ 2. 上海市东方海事工程技术有限公司 上海 200011)
}

\begin{abstract}
摘要: 对盘式永磁调速器建立 $3 \mathrm{D}$ 瞬态浴流场数值模型, 研究永磁调速器在离心负载下的调速性能以及导体盘材料和厚度等 因素对调速性能的影响机理。研究结果表明, 永磁调速器驱动离心负载时, 输出转速和力矩随着气隙非线性变化。在调速过 程中, 存在输出转速与力矩随气隙变化剧烈的区域, 导致调速精度降低。将导体盘材料由铜改成铝可以使调速过程更加平稳。 存在一个铜导体盘厚度值, 对应输出转速和力矩随气隙变化最为剧烈, 调速性能最差。对于调速精度要求高的场所, 选取的 铜导体盘厚度应当远离这个值, 或者选用铝导体盘, 以获得更好的调速性能。
\end{abstract}

关键词: 永磁调速器; 离心负载; 调速性能

中图分类号: TH113

\section{Speed Governing Performance of the Permanent Magnetic Coupler under Centrifugal Load}

\author{
WU Ming $^{1,2}$ MA Yushun ${ }^{1,2}$ KUANG Jun ${ }^{1,2}$ HUANG Xiaobing ${ }^{2}$ \\ (1. China Ship Scientific Research Center, Shanghai 200011; \\ 2. Shanghai Oriental Maritime Engineering Technology Co., Ltd, Shanghai 200011)
}

\begin{abstract}
A numerical model for 3D transient eddy field of the permanent coupler is established. The speed governing performance of the permanent magnetic coupler under the centrifugal load is investigated with this model. Effects of the material and thickness of the conductor disc on the speed governing performance are also studied. The results indicate that the output rotational speed and torque are nonlinear to air gap. The output rotational speed and torque alter sharply with the increase of the air gap in a rotational speed region, therefore reducing the speed governing precision. The substitution of the copper disc by an aluminum disc can improve the speed governing stability of the permanent magnetic coupler. There exists a value of the thickness of the copper disc, under which the output rotational speed and torque alter most intensely with the air gap, and therefore the speed governing performance is the worst. In the situation of demand on high precision of speed governing, the selected copper disc thickness should be away from this value or an aluminum disc should be chosen to achieve better speed governing performance.
\end{abstract}

Key words: permanent magnetic coupler; centrifugal load; speed governing performance

\section{0 前言}

永磁调速器是一种新型柔性传动设备, 主要用 于原与风机等离心负载的节能调速。永磁调速器按 照结构类型可以分成盘式永磁调速器和筒式永磁调 速器两大类, 其中盘式永磁调速器具有结构简单、 安装使用方便等优点, 在工程上应用最为广泛。永 磁调速器具有节能环保、可靠性高、能有效降低振

* 上海张江国家自主创新示范区专项发展资金资助项目 (201505-HPC104-004)。20180619 收到初稿, 20190125 收到修改稿
动、柔性启动等特点, 在工业上得到了广泛应用, 并作为国家重点节能技术被大力推广[1]。

目前国内外研究者对永磁调速器开展了众多研 究, 主要集中在性能研究、结构设计优化以及节能 应用等方面。性能研究主要包括采用层理论 ${ }^{[2]}$ 、等 效磁路法 ${ }^{[3]}$ 、分离变量法 ${ }^{[4]}$ 、应力张量法 ${ }^{[5]}$ 、矢量磁 位法 ${ }^{[6-7]}$ 等理论方法对电磁转矩、浴流损耗、轴向力 等电磁特性进行预测和分析, 以及采用有限元等数 值模拟和试验方法对永磁调速器的电磁特性 ${ }^{[8]}$ 和温 度特性 ${ }^{[9]}$ 等性能进行定量研究和分析。结构优化设 计主要集中在对永磁体占空比、极数和磁化方向、 导体盘厚度等基本结构参数 ${ }^{[10-13]}$ 进行研究和分析, 
以及对冷却结构 ${ }^{[14-15]}$ 和调速机构 ${ }^{[16]}$ 的优化设计与 研究, 同时也包括旋角式永磁调速器 ${ }^{[17]}$ 、混合式高 效永磁调速器 ${ }^{[18]}$ 、双筒型永磁调速器 ${ }^{[19-20]}$ 等一些新 型永磁调速器设计。节能应用主要研究永磁调速器 相比于其他调速技术所具备的技术特点 ${ }^{[21]}$ 以及节 能效益 ${ }^{[22]}$ 。目前针对永磁调速器所开展的研究大多 以永磁调速器作为独立研究对象。对于离心负载, 永磁调速器输出参数与负载运行工况相互耦合, 因 此有必要将永磁调速器与负载设备作为整体系统来 分析永磁调速器的调速性能。WALLACE 等 ${ }^{[23-24]}$ 对 永磁调速器的调速特性进行了试验研究, 分析了它 在风机、泵类负载应用中的节能效果, 然而缺乏对 调速性能影响机理的深入分析与研究。杨超君等 ${ }^{[25]}$ 对永磁调速器调速性能开展了一系列研究, 采用理 论分析、数值模拟和试验相结合的方法研究了永磁 调速器在恒扭矩和离心负载下的调速性能, 然而研 究的转速范围较小, 无法完整反应出整体的调速性 能, 同时也缺乏对调速性能系统的深入分析。目前 永磁调速器在离心负载下的调速性能研究仍然较为 贯乏, 需要进一步深入的定量分析和系统性研究。

本文针对在离心负载下的盘式永磁调速器, 采 用数值模拟方法对永磁调速器在整个调速区间内的 调速性能进行了系统分析, 并研究了导体盘材料、 厚度等因素对调速性能的影响机理, 提出了相应改 善调速性能的方法。

\section{1 模型介绍}

本文所研究的永磁体调速器含有 15 对永磁体。 为了提高计算效率, 在计算过程中仅取永磁调速器 1/15 的区域进行计算。同时只考虑与磁性相关的材 料, 其余材料均作为空气处理。简化后永磁调速器 如图 1 所示: 导体盘和导体盘轭铁组成导体转子, 充磁方向相反的两块永磁体和永磁盘轭铁组成永磁 转子。同时为了考虑端部漏磁效应, 计算区域设置 了一个包含所有部件的大空间。除了结构简化外, 在计算过程中还采用了一些其他主要假设: (1) 所有 材料各向同性, 忽略磁滞效应; (2) 忽略导体盘轭铁 和永磁体轭铁的磁饱和影响; (3) 永磁体为线性材 料, 并且均匀磁化。

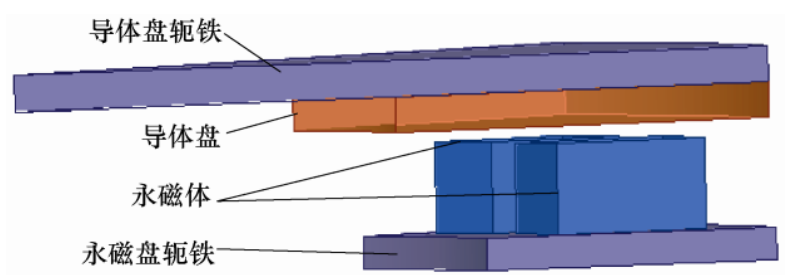

图 1 永磁调速器简化计算模型
$\boldsymbol{A}, \boldsymbol{\varphi}-\boldsymbol{A}$ 方法具有精度高、适用于多连通区域 等优点，是处理浴流场最常用的计算方法之一。尽 管该方法在求解非浴流区域时同样需要计算矢量磁 位, 未知变量多, 计算量较大。但是考虑到本文所 研究计算区域仅取永磁调速器 $1 / 15$ 的区域, 计算量 不大, 因此本文采用 $\boldsymbol{A}, \varphi-\boldsymbol{A}$ 方法求解涡流场。求 解区域分为永磁区 $\Omega_{\mathrm{p}}$ (永磁体)、浴流区 $\Omega_{\mathrm{c}}$ (导体盘、 导体盘轭铁)和空气区 $\Omega_{\mathrm{a}}$ (其他区域)。

永磁区 $\Omega_{\mathrm{p}}$ 基本方程为

$$
\nabla \times \frac{1}{\mu}(\nabla \times \boldsymbol{A})-\nabla\left(\frac{1}{\mu} \nabla \cdot \boldsymbol{A}\right)=\boldsymbol{J}_{\mathrm{s}}
$$

浴流区 $\Omega_{\mathrm{c}}$ 基本方程为

$$
\begin{gathered}
\nabla \times \frac{1}{\mu}(\nabla \times \boldsymbol{A})+\gamma\left[\frac{\partial \boldsymbol{A}}{\partial t}+\nabla \varphi-\boldsymbol{v} \times(\nabla \times \boldsymbol{A})\right]- \\
\nabla\left(\frac{1}{\mu} \nabla \cdot \boldsymbol{A}\right)=0 \\
\nabla \cdot\left\{\gamma\left[\frac{\partial \boldsymbol{A}}{\partial t}+\nabla \varphi-v \times(\nabla \times A)\right]\right\}=0
\end{gathered}
$$

空气区 $\Omega_{\mathrm{a}}$ 基本方程为

$$
\nabla \times \frac{1}{\mu}(\nabla \times \boldsymbol{A})-\nabla\left(\frac{1}{\mu} \nabla \cdot \boldsymbol{A}\right)=0
$$

式中, $\mu$ 为磁导率, $\boldsymbol{A}$ 为矢量磁位, $\boldsymbol{J}_{\mathrm{s}}$ 为永磁区等 效电流密度, $\gamma$ 为电导率, $\varphi$ 为标量电位, $v$ 为线 速度, $t$ 为时间。

对于涡流区 1 与非涡流区 2 的边界 $S_{12}$, 其边界 条件为

$$
\begin{gathered}
\boldsymbol{A}_{1}=\boldsymbol{A}_{2} \\
\frac{1}{\mu_{1}} \nabla \cdot \boldsymbol{A}_{1}=\frac{1}{\mu_{2}} \nabla \cdot \boldsymbol{A}_{2} \\
\boldsymbol{n}_{12} \times \frac{1}{\mu}\left(\nabla \times \boldsymbol{A}_{1}\right)=\boldsymbol{n}_{12} \times \frac{1}{\mu}\left(\nabla \times \boldsymbol{A}_{2}\right) \\
\boldsymbol{n}_{12} \cdot\left(\frac{\partial \boldsymbol{A}_{1}}{\partial t}+\nabla \varphi_{1}-\boldsymbol{v} \times\left(\nabla \times \boldsymbol{A}_{1}\right)\right)=0
\end{gathered}
$$

圆周方向两个对应面 $S_{3}$ 和 $S_{4}$ 为周期性边界条 件，表达式为

$$
\begin{aligned}
\boldsymbol{A}_{3} \cdot \boldsymbol{n}_{3} & =-\boldsymbol{A}_{4} \cdot \boldsymbol{n}_{4} \\
\boldsymbol{A}_{3} \times \boldsymbol{n}_{3} & =-\boldsymbol{A}_{4} \times \boldsymbol{n}_{4} \\
\varphi_{3} & =\varphi_{4}
\end{aligned}
$$

对于计算区域的其他外边界 $S_{5}$, 边界条件为

$$
\begin{gathered}
\boldsymbol{n}_{5} \cdot \boldsymbol{A}=0 \\
\boldsymbol{n}_{5} \times \frac{1}{\mu}(\nabla \times \boldsymbol{A})=0
\end{gathered}
$$

式中, $\boldsymbol{n}_{12}$ 为界面 $S_{12}$ 单位法矢量, $\boldsymbol{n}_{3} 、 \boldsymbol{n}_{4}$ 和 $\boldsymbol{n}_{5}$ 分别 
为外边界面 $S_{3} 、 S_{4}$ 和 $S_{5}$ 的单位外法矢量。

本文采用有限元方法求解上述方程组 ${ }^{[26]}$, 在获 得矢量磁位 $\boldsymbol{A}$ 和标量电位 $\varphi$ 场后, 通过 $\boldsymbol{B}=\nabla \times \boldsymbol{A}$ 可 以获得磁感应强度 $\boldsymbol{B}$; 涡流区的感应电涡流密度 $\boldsymbol{J}$ 可以通过 $\boldsymbol{J}=-\gamma\left[\frac{\partial \boldsymbol{A}}{\partial t}+\nabla \varphi-\boldsymbol{v} \times(\nabla \times \boldsymbol{A})\right]$ 获得, 浴流 损耗 $P$ 可以通过 $P=\int_{V} \frac{|\boldsymbol{J}|^{2}}{\gamma} \mathrm{d} V$ 计算得到。力矩 $T$ 和 轴向力 $F_{z}$ 采用虚功原理计算, 计算式分别为 $T=-\frac{\partial W_{\mathrm{m}}}{\partial \theta}$ 和 $F_{z}=-\frac{\partial W_{\mathrm{m}}}{\partial z}$, 其中 $W_{\mathrm{m}}$ 为磁场能量, $\theta$ 为圆周方向角度, $z$ 为轴向方向。永磁调速器各部 件的尺寸和物性参数见表 1 。

表 1 永磁调速器各部件尺寸和物性参数

\begin{tabular}{|c|c|c|}
\hline 部件 & 性能或尺寸参数 & 数值 \\
\hline \multirow{7}{*}{ 永磁体 } & 材料 & 钕铁硼 $40 \mathrm{H}$ \\
\hline & 相对磁导率 $\mu_{\mathrm{r}}$ & 1.09 \\
\hline & 矫顽力 $H_{\mathrm{c}} /\left(\mathrm{kA} \cdot \mathrm{m}^{-1}\right)$ & 923 \\
\hline & 长 $L / \mathrm{mm}$ & 50 \\
\hline & 宽 $W / \mathrm{mm}$ & 25 \\
\hline & 厚度 $H / \mathrm{mm}$ & 25 \\
\hline & 分布半径 $R / \mathrm{mm}$ & 200 \\
\hline \multirow{6}{*}{ 导体盘 } & 材料 & 铜(铝) \\
\hline & 电导率 $\gamma /\left(\mathrm{S} \cdot \mathrm{m}^{-1}\right)$ & $5.8 \times 10^{7}\left(3.8 \times 10^{7}\right)$ \\
\hline & 相对磁导率 $\mu_{\mathrm{r}}$ & $1(1)$ \\
\hline & 内径 $R_{\mathrm{i}} / \mathrm{mm}$ & 122.5 \\
\hline & 外径 $R_{\mathrm{o}} / \mathrm{mm}$ & 242.5 \\
\hline & 厚度 $H / \mathrm{mm}$ & 10 \\
\hline \multirow{2}{*}{ 轭铁 } & 电导率 $\gamma /\left(\mathrm{S} \cdot \mathrm{m}^{-1}\right)$ & $2 \times 10^{6}$ \\
\hline & 磁导率 $\mu$ & 磁化曲线 ${ }^{[3]}$ \\
\hline \multirow{3}{*}{ 导体盘轭铁 } & 内径 $R_{\mathrm{i}} / \mathrm{mm}$ & 0 \\
\hline & 外径 $R_{\mathrm{o}} / \mathrm{mm}$ & 242.5 \\
\hline & 厚度 $H / \mathrm{mm}$ & 10 \\
\hline \multirow{3}{*}{ 永磁体轭铁 } & 内径 $R_{\mathrm{i}} / \mathrm{mm}$ & 150 \\
\hline & 外径 $R_{\mathrm{o}} / \mathrm{mm}$ & 242.5 \\
\hline & 厚度 $H / \mathrm{mm}$ & 9 \\
\hline
\end{tabular}

\section{2 结果分析}

\section{1 模型验证}

本文首先通过试验对所建立的永磁调速器涡流 场数值模型进行验证。图 2a 所示为永磁调速器在静 止状态下不同气隙对应的轴向力分布, 图 $2 b$ 为永磁 调速器在运转过程中不同转速差对应的输出力矩, 运转过程中气隙固定为 $4 \mathrm{~mm}$ (无特殊说明均为此 值, 此值为实际生产制造过程中综合考虑传递能力 和安装可靠性后选定的最小气隙), 导体盘材料为铜 (无特殊说明均为此材料)。图 2 中实线为试验值,
点画线为模拟值, 带方块实线为模拟值与试验值的 相对误差。图中结果表明, 数值模拟结果与试验结 果吻合良好。因此, 本文建立的数值模型是可靠的。

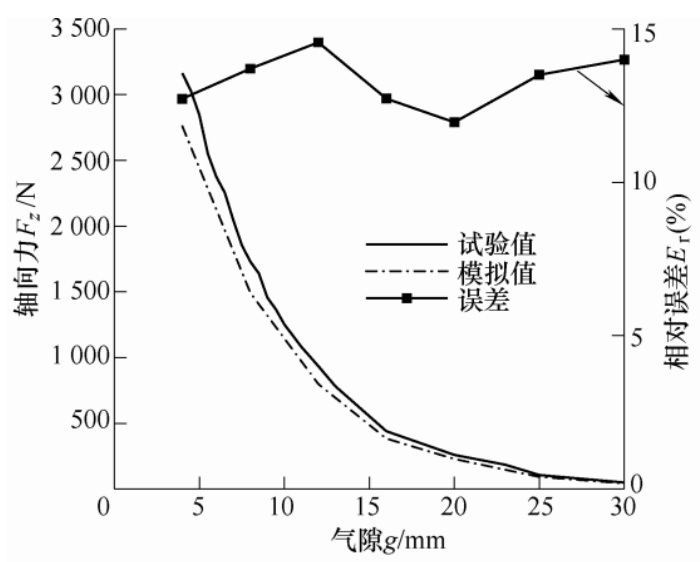

(a) 静态轴向力分布

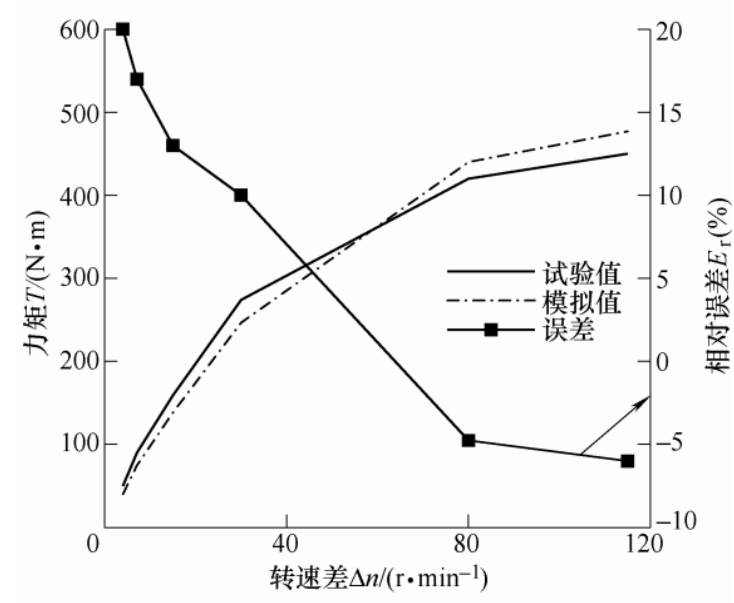

(b) 输出力矩

图 2 永磁调速器数值模型验证

\section{2 永磁调速器输出特性}

图 3 所示为导体转子与永磁转子转速差为 30 $\mathrm{r} / \mathrm{min}$ 、气隙为 $4 \mathrm{~mm}$ 时的磁感应强度矢量分布。从 图 3 中可知, 主磁路为磁力线从一个永磁体出发, 穿过气隙、导体盘进入轭铁, 然后再从另一侧通过 导体盘、气隙进入相邻永磁体, 再通过永磁体底部 的轭铁完成磁力线闭合。大部分的磁力线从主磁路 经过, 同时也存在一部分磁力线并没有穿过导体盘, 而是通过周围气隙等区域直接与自身或者相邻永磁 体构成磁路, 这一部分磁力线属于漏磁部分。随着

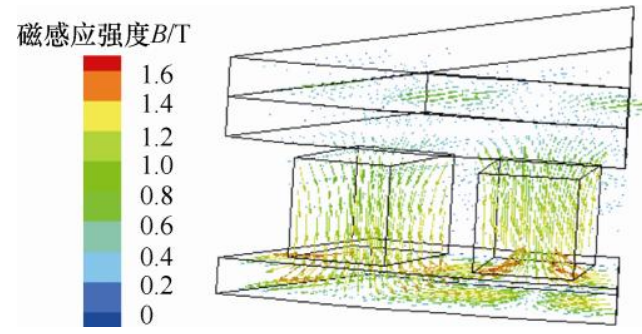

图 3 磁感应强度分布 
气隙的增加, 漏磁量越来越大, 主磁通变小, 从而 导致传递能力发生变化, 这也是永磁调速器的调速 原理。

图 4 所示为铜导体盘上的感应电浴流分布。电 涡流成环形且数目与永磁体数目一致。并且电涡流 中心与永磁体中心在周向上存在角度差。永磁调速 器所传递力矩除了与电浴流强度有关, 同时也与该 角度差有关。环形电涡流可等效成一个永磁体, 当 电浴流中心与永磁体中心很靠近时, 导体盘和永磁 体之间的作用力在周向上的分量会相互抵消，所传 递的力矩也很小。只有当存在一定角度差时, 才能 够传递足够大的力矩。

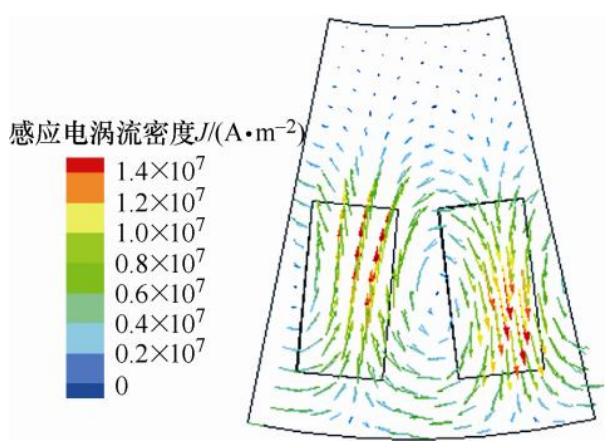

图 4 导体盘电浴流分布

永磁调速器的输出特性是研究其调速性能的基 础。在此先对永磁调速器的输出特性进行研究和分 析。图 5 所示为气隙为 $4 \mathrm{~mm}$ 时力矩随转速差的变 化关系。从图 5 中可知, 在转速差较小时, 力矩随 着转速差的增加而增加, 这是由于导体盘切割磁力 线速度增加, 感应电动势与电流增加, 力矩也相应 增加。当转速差增加到一定值时, 力矩随着转速差 的增加反而减小, 这是由于转速差大时, 导体盘漏 磁感抗作用增加, 导致感应电流变化滞后于磁场变 化, 电涡流中心和永磁体中心的角度差偏离最佳位 置, 从而导致力矩减小; 此外, 随着转速差的增加, 集肤效应更加明显，导体盘有效受力区域减小，从

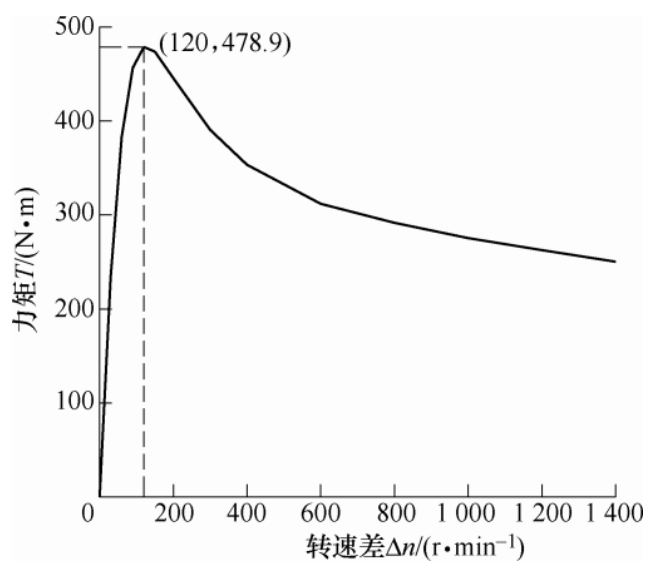

图 5 力矩随转速差分布
而导致力矩减小。当气隙为 $4 \mathrm{~mm}$ 时, 永磁调速器 在转速差为 $120 \mathrm{r} / \mathrm{min}$ 时达到最大扭矩 $478.9 \mathrm{~N} \cdot \mathrm{m}$ 。

\section{3 永磁调速器调速性能}

图 6 所示为导体盘材料为铜时永磁调速器在不 同气隙下对应的特性曲线，曲线附近数字表示气隙 大小。导体转子转速(输入转速)恒定为 $1485 \mathrm{r} / \mathrm{min}$, 横坐标为永磁转子转速(输出转速)。图 6 中点画线 为额定功率 $50 \mathrm{~kW}$ 的离心负载(额定转速 1485 $\mathrm{r} / \mathrm{min})$ 。图 6 中结果表明, 在不同气隙下, 永磁调速 器的力矩随转速变化趋势一致, 均存在一个最大力 矩, 对应的输出转速为临界转速。并且在不同气隙 下, 临界转速非常接近(1 $365 \mathrm{r} / \mathrm{min}$ 左右)。当输出 转速相同时, 力矩随着气隙的增加而减小。气隙越 大，力矩减小程度越缓慢。图 6 中实线和点画线交 点为工况点。由图 6 可知, 在工况点处, 负载斜率 均大于特性曲线斜率，因此工况点都是稳定的。

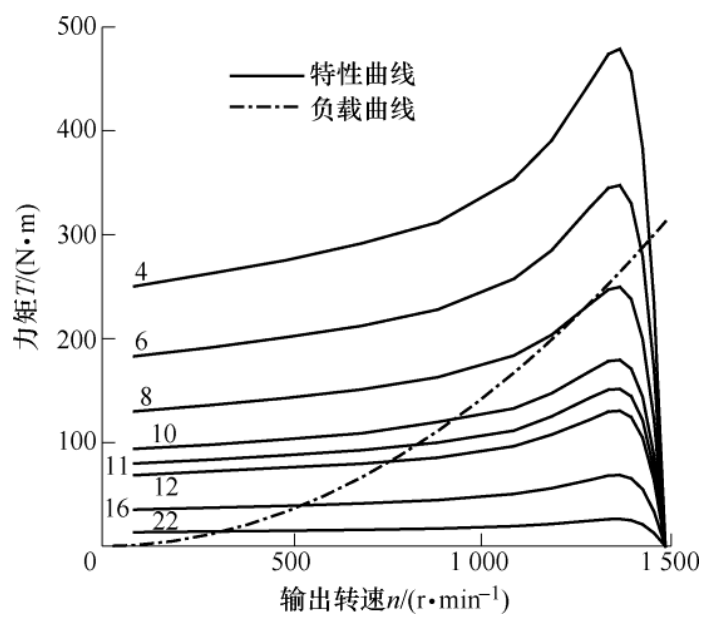

图 6 永磁调速器特性曲线

在泵与风机等离心负载的调速过程中, 输出流 量和压力是重点关注的两个参数。根据离心负载运 行特性可知, 输出流量与输出转速成正比, 输出压 力和力矩均与转速平方成正比。因此, 重点对输出 转速和力矩随气隙的演变规律进行分析。图 7 所示 为在 $50 \mathrm{~kW}$ 离心负载下, 永磁调速器输出转速和 力矩随气隙变化的关系。由图 7 可知, 随着永磁 调速器气隙增加, 输出转速和力矩均减小。但是 图 7 中曲线的斜率并不是定值, 而是随着气隙的 增加, 在某一点处斜率绝对值快速增加到最大值。 当转速差较小时, 输出转速接近输入转速, 此时 工况点在临界转速的右侧, 如图 6 所示, 负载曲 线斜率为正值, 永磁调速器特性曲线斜率为负值, 这两者差值很大; 随着气隙的增加, 输出转速和 力矩缓慢减小, 此阶段调速性能稳定。但是当气 隙增加到 $8 \mathrm{~mm}$ 左右时, 此时工况点在临界转速左 侧, 永磁调速器特性曲线和负载曲线的斜率很接 
近(图 6), 因此输出转速和力矩随着气隙的增加急 剧降低, 此阶段调速性能差。

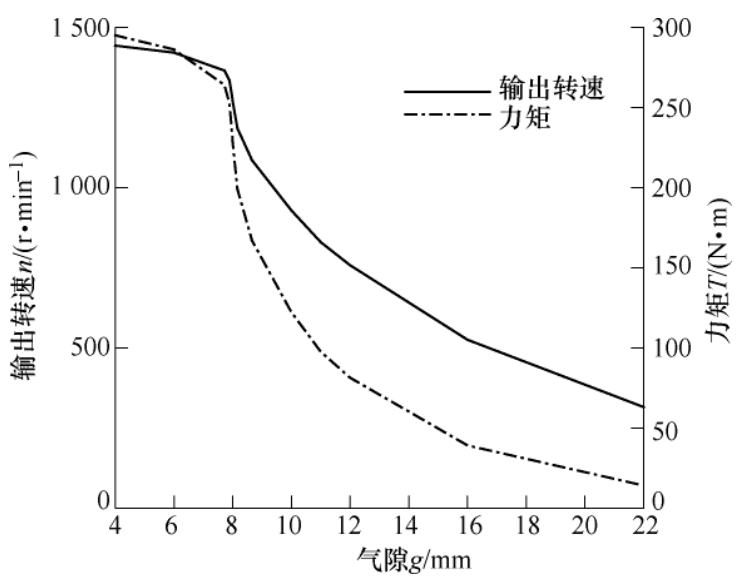

图 7 永磁调速器调速曲线

以输出转速为例, 输出转速从 $1335 \mathrm{r} / \mathrm{min}$ 降低 到 $1185 \mathrm{r} / \mathrm{min}$ 时, 气隙仅增加了 $0.27 \mathrm{~mm}$ 。由于永 磁调速器的气隙调节一般通过机械装置完成, 调节 精度较低。因此在上述转速区间内难以准确调节转 速, 甚至会导致在调速过程中该转速区间被直接跳 过, 无法获得其中的转速。在永磁调速器使用过程 中, 一般会对输出转速或者压力进行监测并以此自 动调节气隙。输出转速随气隙变化过大时, 会导致 无法获得一个精确稳定的转速, 输出转速会在目标 转速附近来回震荡, 从而导致输出压力、流量等发 生震荡, 影响设备、管路的安全使用。因此在实际 使用过程中需要避免这种现象的发生。

\section{4 导体盘材料对调速性能的影响}

为了缓解输出转速和力矩在调速过程中变化过 于剧烈的问题, 可以通过调整永磁调速器输出特性 曲线实现。相对简单方式的是通过改变永磁调速器 导体盘材料来调整永磁调速器的特性曲线。对于导 体盘材料，工程上最常用的是铜和铝。图 8 所示实 线和虚线分别表示导体盘材料为铜和铝时永磁调速 器在不同气隙下的输出特性曲线, 导体盘厚度均为 $10 \mathrm{~mm}$ 。对于不同导体盘材料, 特性曲线的变化趋 势一致。对比相同气隙下的特性曲线可以发现, 采 用不同材料时最大力矩几乎不变，但是最大力矩对 应的临界转速变小。实际上永磁调速器与异步电机 工作原理类似, 都是通过感应产生励磁磁场, 从而 传递力矩。永磁调速器的特性曲线与异步电机特性 曲线也相似。由文献[27]可知, 最大力矩与转子电 阻无关, 但是对应的临界转差率(临界转速对应的转 差率)随导体电阻增加而增加, 临界输出转速相应减 小。从图 8 中可知, 导体盘采用铝后, 特性曲线变 得更加平缓。

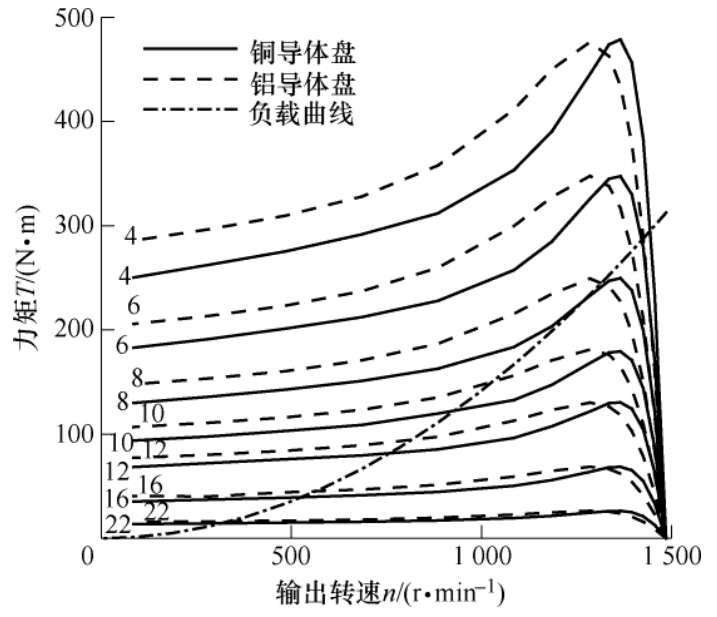

图 8 导体盘材料对特性曲线的影响

由于力矩和输出转速随气隙变化的趋势相似, 为了简便起见, 仅对输出转速随气隙的变化曲线进 行分析。图 9 所示为采用不同导体盘材料时的调速 曲线。对于不同导体盘材料, 其调速曲线变化趋势 相似。但是采用铝材料后, 输出转速变化更加均匀, 曲线斜率绝对值的最大值从 $754.46 \mathrm{r} /(\mathrm{min} \cdot \mathrm{mm})$ 减小 到了 $177.82 \mathrm{r} /(\mathrm{min} \cdot \mathrm{mm})$ 。由此可知, 导体盘采用铝 材料后, 调速性能明显得到改善。但是需要注意的 是采用铝材料后, 在最小气隙 $(4 \mathrm{~mm})$ 下永磁调速器 的输出转速、力矩和功率都减小, 表明永磁调速器 传递能力所有降低。在最大气隙 $(22 \mathrm{~mm})$ 下, 永磁调 速器的输出转速变大, 表明在整个气隙段内永磁调 速器的输出转速区域变窄。

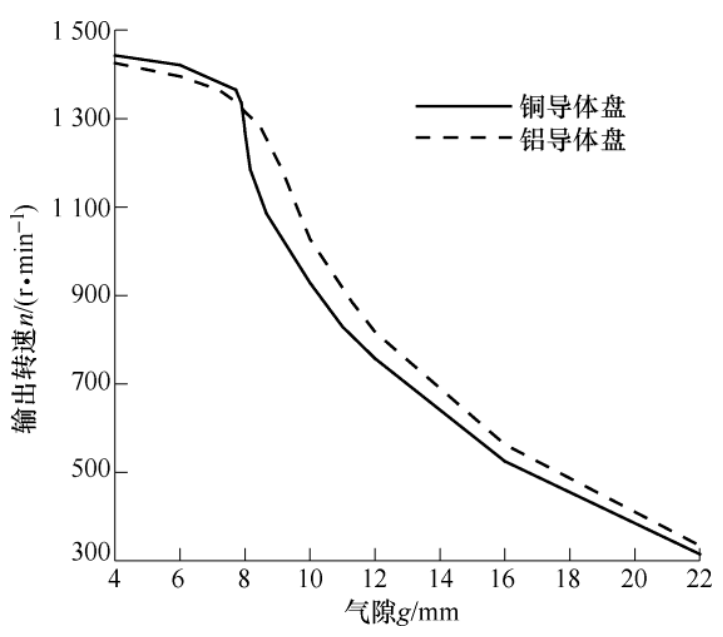

图 9 不同导体盘材料时的调速曲线

\section{5 导体盘厚度对调速性能的影响}

改变导体盘材料, 实际上是改变导体盘的电阻。 除了更换导体盘材料外，改变导体盘的厚度同样会 影响导体盘电阻, 从而达到改变永磁调速器输出特 性和调速性能的目的。本文采用了 $2 \mathrm{~mm} 、 4 \mathrm{~mm}$ 、 $6 \mathrm{~mm} 、 8 \mathrm{~mm} 、 10 \mathrm{~mm}$ 和 $12 \mathrm{~mm}$ 这六组导体盘厚度 值进行分析。图 10 所示为不同导体盘厚度时永磁调 
速器的输出特性曲线。从图 10 中可知, 在相同气隙 下, 随着导体盘厚度的增加, 所传递的最大力矩反 而减小。这是由于最大力矩与导体盘漏磁感抗成反

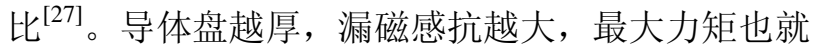
越小。同时, 最大力矩所对应的临界输出转速也随 着导体盘厚度的增加而增加, 这是由于临界转差率 正比于导体盘电阻, 反比于导体盘漏磁感抗。导体 盘厚度增加, 电阻减小, 漏磁感抗增加, 从而导致 临界转差率减小, 临界输出转速增加。

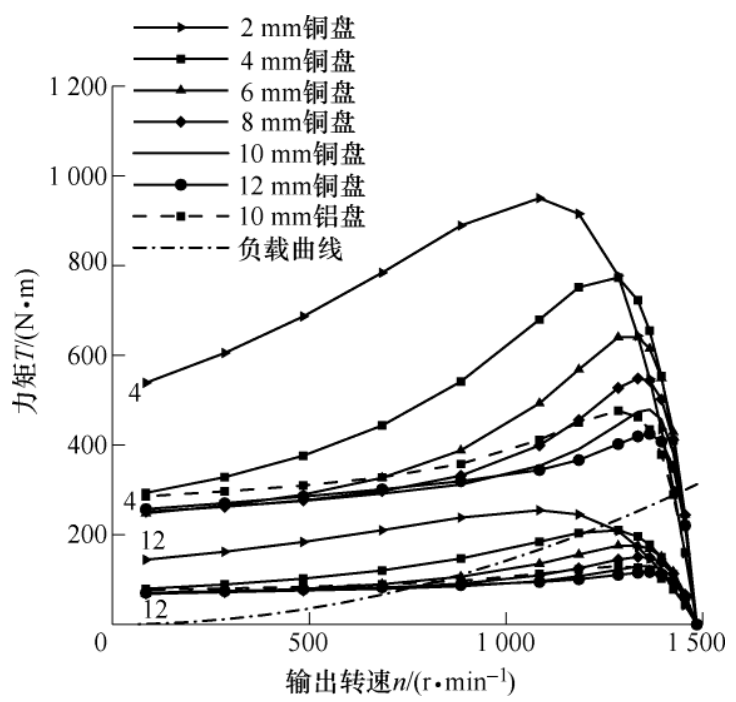

图 10 不同导体盘厚度时的特性曲线

图 11 所示为不同导体盘厚度时的调速曲线。由 图 11 可知, 不同导体盘厚度所对应的调速曲线形状 相似, 但是输出转速随气隙的变化均匀程度明显不 同。当导体盘厚度从 $2 \mathrm{~mm}$ 增加到 $12 \mathrm{~mm}$ 时, 各调 速曲线的斜率绝对值的最大值分别为 124.60 $\mathrm{r} /(\mathrm{min} \cdot \mathrm{mm}) 、 161.94 \mathrm{r} /(\mathrm{min} \cdot \mathrm{mm}) 、 491.41 \mathrm{r} /(\mathrm{min} \cdot \mathrm{mm}) 、$ $1108.37 \mathrm{r} /(\mathrm{min} \cdot \mathrm{mm}) 、 754.46 \mathrm{r} /(\mathrm{min} \cdot \mathrm{mm})$ 和 264.71 $\mathrm{r} /(\mathrm{min} \cdot \mathrm{mm})$ 。并且输出转速变化最剧烈的地方随着 导体盘厚度的增加而下移, 这是与图 10 中临界输出 转速的变化趋势对应的。由以上结果可知, 导体盘

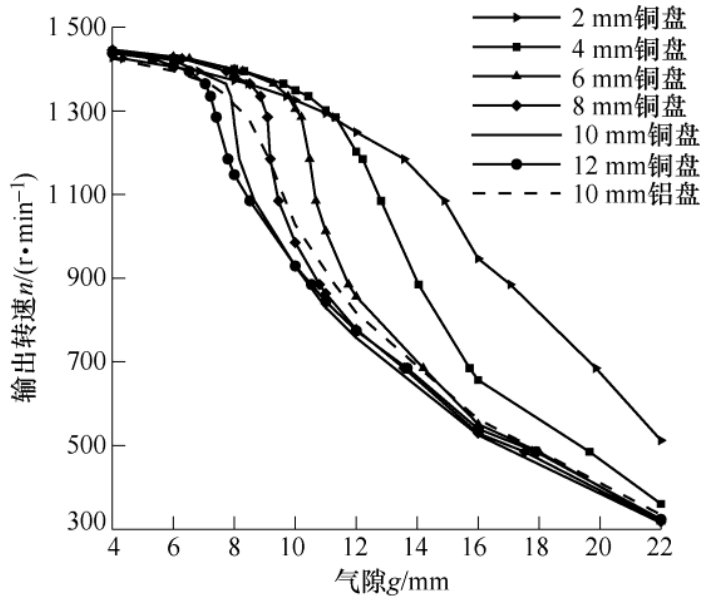

图 11 不同导体盘厚度时的调速曲线
厚度为 $8 \mathrm{~mm}$ 时, 调速曲线斜率绝对值的最大值比 其他导体盘厚度下对应的值更大，调速曲线变化最 不均匀, 调速性能最差。导体盘厚度越远离这个值, 输出转速变化更加均匀, 调速性能越好。但是需要 注意的是在最小气隙下的输出转速和力矩也相应减 小, 传递能力降低, 并且整个气隙段对应的输出转 速区域也相应变小。因此, 在实际使用过程中, 可 以通过合理增加或者减小导体盘厚度来改善永磁调 速器调速性能。

对比图 11 中采用 $2 \mathrm{~mm}$ 铜导体盘和 $10 \mathrm{~mm}$ 铝 导体盘这两种相对最好的情况可知, 采用 $2 \mathrm{~mm}$ 厚 铜导体盘时调速曲线的斜率绝对值的最大值更小， 整个调速过程更加平稳。在最小气隙 $4 \mathrm{~mm}$ 的情况 下, 两者的输出转速和功率差异很小, 最大传递能 力接近。但是在最大气隙 $22 \mathrm{~mm}$ 下, 导体盘采用 2 $\mathrm{mm}$ 铜盘的永磁调速器输出转速更高, 表明调速区 域更窄。在实际永磁调速器选型过程中, 应该综合 考虑调速性能平稳性、传递能力及调速区域大小等 因素的影响。

\section{3 结论}

(1) 通过数值模拟的方法, 对盘式永磁调速器 驱动离心负载时的调速性能开展了研究。结果表明 在调速过程中, 存在输出转速与力矩随气隙变化剧 烈的区域，导致调速精度降低。

（2）相比于铜导体盘，采用铝导体盘可以使调 速过程更平稳, 调速性能更好。

(3) 存在一个铜导体盘厚度值, 对应输出转速 和力矩随气隙变化最为剧烈, 调速精度最差。对于 调速精度要求高的场所, 选取的铜盘厚度应当远离 此值, 以获得更好的调速性能。

（4）通过改变铜导体盘厚度或者采用铝导体盘 改善永磁调速在离心负载下的调速性能时, 往往会 导致永磁调速器最大传递能力降低以及输出转速区 间变小。在永磁调速器实际使用过程中, 应该综合 考虑这些因素来合理选取参数。

\section{参 考 文 献}

[1] 中华人民共和国工业和信息化部. 国家重点推广的电 机节能先进技术目录(第一批)[J]. 中国设备工程, 2014(8): 71-72.

Ministry of Industry and Information Technology of the People's Republic of China. National motor energy-saving advanced technology directory (first batch) $[\mathrm{J}]$. China Plant Engineering, 2014(8): 71-72. 
[2] SMITH A C, WILLSAMSON H, BENHAMA N, et al. Magnetic drive couplings[C]//Electrical Machines and Drives. 9th International Conference on Electrical Machines and Drives, September 1-3, 1999, Canterbury, UK. Canterbury: IET, 1999: 232-236.

[3] MOHAMMADI S, MIRSALIM M, VAEZ-ZADEH S, et al. Analytical modeling and analysis of axial-flux interior permanent-magnet couplers[J]. IEEE Transactions on Industrial Electronics， 2014，61(11): 5940-5947.

[4] CANOVA A, VUSINI B. Analytical modeling of rotating eddy-current couplers[J]. IEEE Transactions on Magnetics, 2005, 41(1): 24-35.

[5] WALLACE A, JOUANNE A V, WILLIAMSON S, et al. Performance prediction and test of adjustable , permanent-magnet, load transmission systems[C]// Industry Applications Conference. Thirty-Sixth IAS Annual Meeting, September 30- October 4, 2001 , Chicago, IL, USA. Chicago: IEEE, 2001(3): 1648-1655.

[6] CHOI J Y, JANG S M. Analytical magnetic torque calculations and experimental testing of radial flux permanent magnet-type eddy current brakes[J]. Journal of Applied Physics, 2012, 111(7): 1-3.

[7] LUBIN T, REZZOUG A. Steady-state and transient performance of axial-field eddy-current coupling[J]. IEEE Transactions on Industrial Electronics, 2015, 62(4): 2287-2296.

[8] 杨超君, 管春松, 丁否, 等. 盘式异步磁力联轴器传动 特性 [J]. 机械工程学报，2014，50(1)：76-84.

YANG Chaojun, GUAN Chunsong, DING Lei, et al. Transmission characteristics of axial asynchronous permanent magnet couplings[J]. Journal of Mechanical Engineering, 2014, 50(1): 76-84.

[9] 杨超君, 周曰华, 李志宝. 鼠笼异步磁力联轴器三维温 度场有限元分析 $[J]$. 机械设计与研究, 2013，29(1): 25-28.

YANG Chaojun, ZHOU Yuehua, LI Zhibao. Analysis on 3-D temperature field of squirrel-cage asynchronous magnetic coupling[J]. Machine Design and Research, 2013, 29(1): 25-28.

[10] 杨超君, 徐燕飞, 孔令营, 等. 调磁式异步磁力联轴器 Halbach 阵列结构磁路有限元分析 [J]. 磁性材料及器 件, 2014，45(3): 5-9.

YANG Chaojun, XU Yanfei, KONG Lingying, et al. Field-modulated asynchronous magnetic coupling Halbach array finite element analysis output torque[J]. Journal of Magnetic Materials and Devices, 2014, 45(3): 5-9.

[11] CHA H R, CHO H W, LEE S H. The influence of magnetization pattern on the performance of permanent magnet eddy current couplings and brakes[J]. Journal of
Electrical Engineering \& Technology, 2008, 3(3): 379-384.

[12] 李桃, 林鹤云, 黄允凯, 等. 基于三维运动浴流场分析 的永磁涡流联轴器特性[J]. 东南大学学报, 2010, 40(2): 301-305.

LI Tao, LIN Heyun, HUANG Yunkai, et al. Characteristic study of permanent magnet eddy current coupling based on 3D moving eddy current field analysis[J]. Journal of Southeast University, 2010, 40(2): 301-305.

[13] 杨超君, 郑武, 李志宝. 可调速异步盘式磁力联轴器的 转矩计算及其影响因素分析 $[\mathrm{J}]$. 电机与控制学报, 2012, 16(1): 85-91.

YANG Chaojun, ZHENG Wu, LI Zhibao. Torque calculation of speed-adjustable asynchronous disk type of magnetic coupling and its impact factors analysis[J]. Electric Machines and Control, 2012， 16(1): 85-91.

[14] 王延杰. 永磁调速器的冷却技术研究[D]. 西安: 西安石 油大学, 2015.

WANG Yanjie. Study on the cooling technology of permanent magnet governor[D]. Xi'an: Xi'an Shiyou University, 2015.

[15] 贺彦霖. 永磁调速器的雾化冷却技术研究[D]. 西安: 西 安石油大学, 2017.

HE Yanlin. The study of the spray cooling on permanent magnet governor[D]. Xi'an: Xi'an Shiyou University, 2017.

[16] 罗永金金. 筒式调速异步磁力耦合器动态性能分析与调 速机构设计 [D]. 镇江: 江苏大学, 2015 .

LUO Yongxin. Dynamic performance analysis and adjustable-speed mechanism design of drum-type adjustable-speed asynchronous magnetic couplers[D]. Zhenjiang: Jiangsu University, 2015.

[17] 高丹. 旋角式永磁调速器电磁场耦合方式、拓扑结构与 实验研究[D]. 沈阳：沈阳工业大学, 2017.

GAO Dan. Research on electromagnetic coupling mode, topology and experiment of the swing angle type permanent adjustable speed device[D]. Shenyang : Shenyang University of Technology, 2017.

[18] 刘岩, 高庆忠, 王森, 等. 新型混合式高效永磁调速器 温度场仿真与分析 $[J]$. 微特电机, 2018，46(2): 69-72. LIU Yan, GAO Qingzhong, WANG Sen, et al. Simulation and analysis of temperature field of new hybrid high-efficiency adjustable permanent magnetic coupler[J]. Small \& Special Electrical Machines, 2018, 46(2): 69-72.

[19] 尹新权, 张亚萍, 王珺. 双筒型永磁调速器的设计与研 究[J]. 矿山机械, 2017，45(12): 59-63.

YIN Xinquan, ZHANG Yaping, WANG Jun. Design and research on a dual-drum permanent magnetic speed 
controller[J]. Mining \& Processing Equipment, 2017, 45(12): 59-63.

[20] MOHAMMADI S , MIRSALIM M. Double-sided permanent-magnet radial-flux eddy current couplers : three-dimensional analytical modelling, static and transient study, and sensitivity analysis[J]. IET Electric Power Applications， 2013， 7(9): 665-679.

[21] 侯剑雄, 杨群发, 何洤标. 大型凝结水洜采用永磁调速 与高压变频技术改造效果比较 $[\mathrm{J}]$. 中国电力, 2015 , 48(8): 135-140.

HOU Jianxiong, YANG Qunfa, HE Ganbiao. Comparative study on effects of large condensate pump improvement with permanent magnet speeding and high-voltage frequency conversion technologies[J]. Electric Power, 2015, 48(8): 135-140.

[22] 赵勇, 张春杰, 王刚, 等. 电厂闭式水洜永磁调速器改 造的经济效益分析 [J]. 华电技术，2015，37(3): 38-41. ZHAO Yong, ZHANG Chunjie, WANG Gang, et al. Analysis on economic benefit of reformation of permanent magnet speed controller of closed water pump in a power plant[J]. Hua Dian Technology, 2015, 37(3): 38-41.

[23] WALLACE A, JOUANNE A V. Industrial speed control: are PM couplings an alternative to VFDs?[J]. IEEE Industry Applications Magazine, 2001, 7(5): 57-63.
[24] WALLACE A, VON JOUANNE A, JEFFRYES R, et al Comparison testing of an adjustable-speed permanentmagnet eddy-current coupling[C]//2000 Annual Pulp and Paper Industry Technical Conference，June 19-23，2000, Atlanta, USA. Atlanta: IEEE, 2000: 73-78.

[25] 吉城龙. 调速型筒式异步磁力耦合器传动性能与调速 性能研究[D]. 镇江: 江苏大学, 2016 .

JI Chenglong. Study on transmission performance and speed-control performance of drum-type speed-control asynchronous magnetic couplers[D]. Zhenjiang: Jiangsu University, 2016.

[26] 周克定. 工程电磁场数值计算理论方法及应用 [M]. 北 京: 高等教育出版社, 1994.

ZHOU Keding. Numerical calculation method and application of engineering electromagnetic field[M]. Beijing: Higher Education Press, 1994.

[27] 邓星钟. 机电传动控制 [M]. 3 版. 武汉: 华中科技大学 出版社, 2001.

DENG Xingzhong. Mechanical \& electrical transmission control[M]. 3rd ed. Wuhan: Huazhong University of Science and Technology Press, 2001.

作者简介: 吴明, 男, 1989 年出生, 博士。主要研究方向为永磁装备的 研发与应用。

E-mail: wumingxjtu@163.com 